\title{
Qualidade e consumo do leite bovino do Vale do Ribeira
}

\section{Cattle milk consumption and quality of the Ribeira Valley \\ Consumo e calidad del leche de vacuno del Valle de la Ribeira}

\author{
Masahiko $\mathrm{Ohi}^{2}$ \\ Ana Carolina Gurgel Knopki ${ }^{1}$ \\ Clarissa Holanda Ramires ${ }^{1}$ \\ Daiane Sanchuki ${ }^{1}$ \\ Franciela Bednarski ${ }^{1}$ \\ Ligia Valéria Nascimento ${ }^{1}$ \\ Lilian Barbosa da Silva ${ }^{1}$ \\ Maria Emília de Miranda ${ }^{1}$ \\ Samuel Fonseca Grotta ${ }^{1}$ \\ Paulo Roberto Dalsenter ${ }^{3}$ \\ Helena Cristina da Silva de Assis $^{3}$ \\ José Francisco Wart ${ }^{3}$ \\ Annelise Kloss ${ }^{4}$
}

\begin{abstract}
RESUMO
Preocupados com o perfil de consumo de leite na região do Vale do Ribeira/PR, o Projeto pertencente ao Programa Universidade Sem Fronteiras da SETI/PR em parceria com o PROLAC - Projeto Leite, Alimento de Criança, objetivou melhorar a qualidade do leite bovino e analisar o perfil do consumo de leite e derivados da população dessa Região. Para alcançar os objetivos foram realizadas visitas em 39 propriedades rurais, constatando problemas higiênico-sanitários e corrigindo-os. Isso resultou na melhora de 33\% na produção de leite e consideráveis melhorias na qualidade. Também, como ferramentas de ação, foram ministradas palestras em escolas municipais e estaduais de nível fundamental e médio, para análise do perfil do consumo de leite dos alunos da região. Todos os alunos de Adrianópolis (100\%) com até 10 anos relatam ingerir 2 copos leite/dia, enquanto 30,3\% dos alunos de Bocaiúva de Sul, com esta faixa etária, consomem quantidade inferior à recomendada por nutricionistas. Importante parcela de indivíduos (40,89\% em Adrianópolis e 21,3\% em Bocaiúva do Sul) consome derivados de origem caseira e artesanal, sem garantias higiênico-sanitárias. O consumo de leite diário é inferior ao recomendado pelo National Institute of Health para crianças de até 12 anos, que é de aproximadamente 4 a 5 copos leite/dia. Faz-se necessária uma intensa ação extensionista para conscientizar o produtor da importância de melhorar a qualidade produtiva e estimular o consumo de leite e derivados oriundos dessas propriedades.
\end{abstract}

Palavras-chave: consumo; leite; produção; qualidade.

\begin{abstract}
Worried about the profile of milk consumption in the region of the Vale do Ribeira/PR, the pertaining Project of the Program Universidade sem Fronteiras from SETI/PR, in partnership with the PROLAC - Projeto Leite, Alimento de Criança, targeted to improve the quality of bovine milk and to analyze the profile of the milk consumption and derivatives of the population of the Region. To reach the objectives, visits in 39 country properties had been carried through, evidencing hygienical-sanitary problems, wich were solved. This re-
\end{abstract}

\footnotetext{
Bolsistas profissionais SETI.

2 Professor orientador UFPR.

3 Professor colaborador UFPR.

4 Bolsista de graduação SETI.
} 
sulted in the improvement of $33 \%$ in the production of milk and considerable improvements in the quality of this milk. Also, as action tools, lectures in municipal and state elementary and high schools been given, to an analysis of the profile of milk consumption of the pupils from the region. All the pupils of Adrianópolis $(100 \%)$ under 10 years old reported that they ingested 2 cupsoper day, while $30.3 \%$ of the children from Bocaiúva do Sul uder that age consumed an inferior amount i relation to the recommended nutritionists. Some important parcel of individuals $(40.89 \%$ in Adrianópolis and $21.3 \%$ in Bocaiúva do Sul) consumes dairy products from homemade and artisanal origin, without hygienical-sanitary guarantees. The daily milk consumption is inferior to what is recommended byf the National Institute of Health for children up to 12 years old, that is approximately 4-5 cups of milk per day. The continuation of that work becomes necessary to the agricultural producer's awareness of the importance in improving the productive quality of milk and stimulating the consumption of milk and dairy products which come from those properties.

Keywords: consumption; Milk; Production; quality.

\section{RESUMEN}

Preocupados con el perfil del consumo de leche en la región de Vale do Ribeira/PR el proyecto perteneciente al programa "Universidade Sem Fronteras" de la SETI/PR en sociedad con el PROLAC - Projeto Leite, Alimento de Criança, decidió mejorar la calidad de leche bovina y analizar el perfil del consumo de leche y derivados de la población de la región. Para alcanzar los objetivos fueron realizadas visitas en 39 propiedades rurales, evidenciando los problemas higiénico-sanitarios siendo estos corregidos. Resulto en la mejora de $33 \%$ en la producción de leche y considerables mejorías en la calidad de leche. También, como herramienta de la acción, fueron dadas conferencias en escuelas municipales y estatales de primaria y secundaria, para el análisis del perfil de la consumición de leche de los alumnos de la región. Todos los alumnos de Adrianópolis (100\%) con hasta 10 años dicen injerir 2 vasos de la leche por día, mientras que $30.3 \%$ de los alumnos de Bocaiúva do Sul con esta venda del etária consumen cantidad inferior recomendada para los nutricionistas. Importantes cantidad de individuos (40.89\% en Adrianópolis y 21.3\% en Bocaiúva del Sul) consume derivados de origen casera y artesanal, sin garantías higiénicas y sanitarias. El consumo de leche es inferior al recomendado por el "National Institute of Heath" (Instituto Nacional de la Salud), para los niños de hasta 12 años, siendo aproximadamente 4 y 5 vasos de leche por día. Hace necesaria la continuación del trabajo para concientizar el producto rural de la importancia para mejorar la calidad de leche producida y estimular el consumo de leche y derivados de la derivación de estas características.

Palabras-clave: consumo; leche; producción; calidad.

Introdução

No século $\mathrm{XX}$, pesquisadores determinaram que o leite bovino possuísse a maioria dos elementos essenciais - micronutrientes, aminoácidos e ácidos graxos essenciais - em quantidades maiores do que em qualquer outro produto isolado. Esse achado permanece irrefutável até os dias de hoje. Além disso, o leite bovino contém proteínas de alta qualidade. Mas, além de conter os nutrientes fundamentais, contém outras substâncias bioativas - enzimas, fatores de crescimento, hormônios e citocinas (moléculas parecidas com os hormônios) -, componentes que reforçam a importância desse alimento para a saúde humana.
Segundo as recomendações do Ministério da Saúde, o consumo de leite, na forma fluida ou de derivados lácteos, varia de acordo com a idade das pessoas. Segundo Lerner et al. (2000) a recomendação para crianças de até dez anos é de $400 \mathrm{ml} /$ dia, isto é, 146 litros/ano de leite fluido ou equivalente na forma de derivados. Para os jovens de 11 a 19 anos, o consumo é maior, de $700 \mathrm{ml} /$ dia ou 256 litros/ano e para os adultos acima de 20 anos a recomendação é de $600 \mathrm{ml} /$ dia ou 219 litros/ano, inclusive para os idosos.

Nota-se que a disponibilidade atual de leite no Brasil ainda não é suficiente para abastecer o mínimo recomendado pelo Ministério da Saúde. Se for considerado o consumo per capita de $600 \mathrm{ml} / \mathrm{dia}$, a dispo- 
nibilidade anual de leite deveria ser de 38 bilhões de litros, muito além do que é produzido.

De acordo com Brito (2009), os dados do IBGE indicam que a produção no País subiu de 18,5 bilhões de litros em 1996 para 24,5 bilhões em 2005, com uma média de 3,3\% ao ano. Carvalho (2007) ainda afirma que o país ocupa uma posição mundial ruim no âmbito da produtividade, que é quase três vezes inferior a da Nova Zelândia e sete vezes menor que a dos Estados Unidos. Tal situação ilustra a necessidade de expansão da produtividade no Brasil, na esteira de melhoramento genético do rebanho, maior profissionalização na gestão das propriedades rurais, melhorias no manejo e na nutrição do rebanho bovino.

No Brasil o leite é produzido, em grande volume, através de um pequeno número de produtores, e por um grande grupo de produtores com um pequeno volume de produção, menos de 50 litros por dia (KRUG, 2000). Nesse sentido, o apoio produtivo à agricultura familiar é visto como um mecanismo de autopromoção da segurança alimentar, melhorando a qualidade e obtendo benefícios por conta disso. Segundo Abramovay (2000), isso significa que a conscientização dos produtores de leite no meio rural deve ser integrada com incentivos técnicos, financeiros e organizacionais para fortalecer a produção dos agricultores familiares, promovendo melhor qualidade do produto e da segurança alimentar.

A cadeia produtiva do leite exige de seus integrantes um gerenciamento qualificado para bem atender e satisfazer o mercado. Essas exigências se baseiam na qualidade da matériaprima, através do controle sanitário, com atenção especial aos problemas de enfermidades infecciosas, carenciais e parasitárias do rebanho; controle higiênico/sanitário do leite cru e resfriado; rígido controle no transporte e na recepção nas unidades de recebimento e nos processos industriais para a transformação do leite em subprodutos aliados aos índices de qualidade (TRONCO, 2000).

O Estado do Paraná tem se destacado no cenário nacional sendo referência na pecuária leiteira em termos de tecnologia, controle e produção (OLIVEIRA, 1997). Existem regiões no estado que não são compatíveis com as características apresentadas acima. Uma das áreas de menor produção leiteira é a região do Vale do Ribeira no Estado do Paraná, caracterizada por apresentar relevo fortemente acidentado - ondulado e montanhoso - e solo rochoso dificultando a atividade de subsistência. A região de Adrianópolis e a região norte de Cerro Azul, situados no Vale, são as que apresentam melhores condições de clima, solo e relevo para desenvolvimento da pecuária leiteira.

O baixo desempenho dos rebanhos leiteiros, nessa região, deve-se a vários fatores, entre eles podemos citar: área inapropriada para o desenvolvimento da atividade, falta de tecnologia adequada, alimentação e manejo alimentar precários, potencial genético inadequado dos rebanhos, pouca capacitação dos produtores para gerenciamento das propriedades associado ao baixo desenvolvimento humano, sendo considerada estatisticamente a região do Estado do Paraná com menor Índice de Desenvolvimento Humano. Outro fator relevante: a falta de conhecimento técnico, ajustado com a realidade social e econômica dos produtores, pode gerar sistemas de produção incompatível com a realidade apresentada. Como sequela, provoca ausência de competitividade com as condições de mercado globalizado, principalmente por esses produtores não apresentarem sustentabilidade, levando-os à falência ou à baixa qualidade de vida $e$, como consequência, advêm a degradação do meio ambiente, o desemprego e o êxodo rural.

Por esses e outros o Projeto "Cuidando da Qualidade do Leite Bovino no Vale do Ribeira/ PR", do Programa Universidade Sem Fronteiras, 
da Secretaria de Estado do Paraná de Ciência, Tecnologia e Ensino Superior, contribui para a ampliação da produtividade leiteira e estimula o consumo de leite de qualidade.

\section{Desenvolvimento e demonstração dos resultados}

O Projeto concentrou suas atividades na cidade de Adrianópolis, atuando de duas formas distintas: visitas e orientação aos produtores rurais associados ao laticínio no município de Adrianópolis - COPLAR (Cooperativa dos Produtores de Leite do Alto Vale do Ribeira) - e, simultaneamente, apresentações de palestras para alunos de escolas municipais e estaduais.

Em uma das vertentes o projeto realizou três visitas a 39 propriedades que abastecem o laticínio e atuou, primeiramente, no conhecimento dos produtores de leite.

A primeira visita consistiu na realização de um diagnóstico de situação, objetivando a busca de informações. Ao diagnosticar as propriedades, foram elaborados cadastros onde se identificaram alguns problemas referentes à qualidade do leite e ao manejo geral do rebanho. Através desse formulário, foram avaliadas as condições de manejo de ordenha e detecção de pontos críticos para estabelecimento de estratégias de correção das deficiências.

O processo de resfriamento se realiza em resfriadores comunitários, estrategicamente distribuídos na região, além dos resfriadores particulares. Entre os 39 produtores, 45\% ainda utilizam o tanque de resfriamento por imersão, método no qual a temperatura ideal de refrigeração deveria ser de aproximadamente $5^{\circ} \mathrm{C}$, e nem sempre é atingida, o que prejudica a qualidade do leite. A ordenha é manual em $87 \%$ das propriedades, prejudicando alguns aspectos sanitários do rebanho.
A falta de preocupação em relação aos aspectos genéticos é detectada no Vale do Ribeira. Aproximadamente $67 \%$ dos produtores não realizam nenhum tipo de seleção de animais, os demais produtores selecionam por aptidão leiteira e poucos por características genéticas e fenotípicas. A produtividade média vaca/dia é de $4,70 \mathrm{~kg}$, uma vez que grande parte do rebanho é composta por animais mestiços com gado de corte o que propicia numa redução da produção leiteira. Somente $10 \%$ dos produtores utilizam a técnica de inseminação artificial, que poderia ser um auxiliar no ganho genético do rebanho.

O controle zootécnico, realizado em $67 \%$ das propriedades, é apenas um controle superficial, o qual não auxilia no gerenciamento e na tomada de decisões.

Na segunda etapa, baseando-se nos resultados obtidos na primeira visita, foi montado um cronograma no qual cada propriedade recebeu uma visita de caráter prático, com o intuito de demonstrar uma ordenha higiênica, abordando os procedimentos recomendados nos momentos anteriores, durante e posteriores a ordenha, ou seja, desde a limpeza dos tetos dos animais, passando por uma correta lavagem dos equipamentos de ordenha, do tanque de refrigeração e local onde é realizada a ordenha. Após a demonstração, foi entregue um kit para o produtor, contendo uma raquete e reagente químico, para diagnóstico de mastite subclínica (CMT), uma caneca telada, para diagnóstico preventivo, e produtos para limpeza dos tetos (pré e pós-dipping). O uso desse kit foi demonstrado durante a ordenha. Além da utilização do "kit" para prevenção e diagnóstico de mastite, o conceito de ordenha higiênica abrange também a higiene no local, que também era inadequada.

A terceira visita teve por finalidade, verificar se os produtores adotaram as técnicas recomendadas, avaliar os impactos que elas surtiram na produtividade e qualidade do leite e reforçar as teorias e práticas já demonstradas. 
Verificou-se que, somente $30 \%$ dos produtores adotaram todos os passos de uma ordenha higiênica. O CMT foi o método mais utilizado pela maioria dos produtores, sendo o percentual de adesão a esta técnica de $81 \%$. A caneca telada é utilizada por $71 \%$ dos produtores e somente $42 \%$ utilizam produtos sanitizantes antes e após a ordenha.

A execução das etapas, acima descritas, propiciou o alcance do objetivo ao constatar a melhora da qualidade do leite produzido e aumento em $33 \%$ na produção.

Tais números demonstram que ainda há necessidade de sensibilização da maioria dos produtores na adoção de técnicas de higiene na ordenha para obtenção de leite com qualidade.

Simultaneamente a essas ações de campo, foram ministradas palestras em escolas municipais e estaduais, com o intuito de sensibilizar a população local quanto ao consumo e produção de leite com qualidade, livre de resíduos químicos e biológicos nocivos para a saúde. O conhecimento adquirido deve ser levado aos pais por esta geração, sendo estes produtores rurais ou não. Durante a realização das palestras, foram distribuídas cartilhas informativas e aplicado um questionário sobre consumo de leite e derivados, origem do leite consumido, bem como a idade e escolaridade dos alunos.

As palestras contemplaram 837 alunos do Ensino Fundamental e Médio de escolas municipais e estaduais.

Constatou-se que os alunos de Adrianópolis com até 10 anos de idade consomem em torno de 2 copos de leite por dia (Tabela 1) e em Bocaiúva do Sul 30,3\% consomem menos que o recomendado pelo National Institute of Health, sendo $1200-1500 \mathrm{mg} /$ dia de cálcio, correspondente a 4 a 5 copos de leite para suprir as necessidades diárias (ZOCCAL, 2009). Apesar de ser uma região produtora de leite, $52 \%$ do leite consumido provém do mercado. A maioria dos alunos - 82,4\% - afirma ferver o leite antes de ingerir. Foi verificado que uma parcela de 40,89\% indivíduos de Adrianópolis e 21,3\% em Bocaiúva do Sul consomem derivados de origem caseira e artesanal, sem garantias higiênico-sanitárias, havendo maior risco de contaminação.

\section{TABELA 1 - CONSUMO DE LEITE EM ADRIANÓPOLIS, POR FAIXA ETÁRIA}

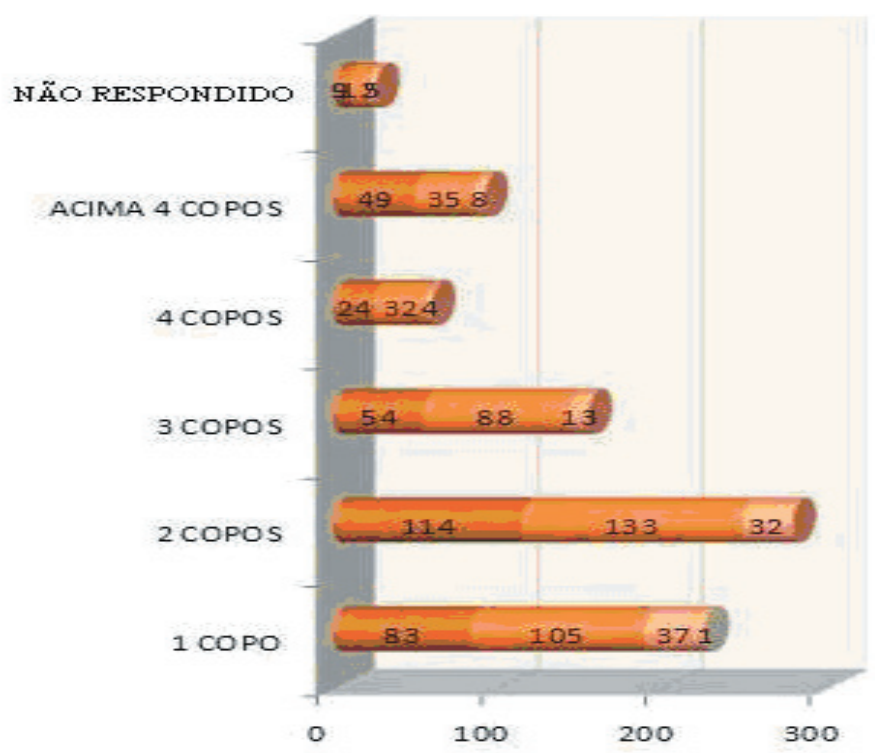

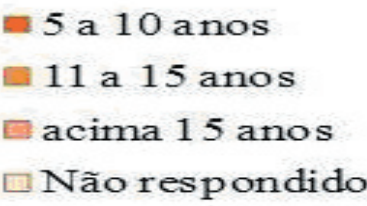

5 a 10 anos

acima 15 anos

Não respondido 


\section{Conclusão}

Considerando os resultados obtidos pelo Projeto Cuidando da Qualidade do Leite Bovino do Vale do Ribeira, verifica-se pequena melhora na qualidade e quantidade de leite oriundo de propriedades assistidas pelo Projeto. Porém, essa melhora pode ser considerada ínfima, em relação aos objetivos iniciais.

Ainda se faz necessária a orientação das crianças e adolescentes quanto à importância do consumo de leite e derivados em quantidade suficiente para um desenvolvimento corporal adequado. Porém, esses alimentos ingeridos

\section{REFERÊNCIAS}

ABRAMOVAY, R. Paradigmas do capitalismo agrário em questão. São Paulo: Hucitec, 2000.

AMERICAN COUNCIL ON SCIENCE AND HEALTH. O leite de vaca e a saúde humana - a verdade. Disponível em: <http://www.lincx.com.br/lincx/saude_a_z/alimentos/ leite_vaca.asp>. Acesso em: 24/3/2009.

BRITO, P. Agricultores familiares de SC investem em fábrica de leite em pó. Disponível em: <http: //www.agrosoft.org.br/agropag/209191.htm>. Acesso em: 20/3/2009.

CARVALHO, G. Competitividade além do leite. Revista Eletrônica Panorama do Leite On line, ano 2, n. 13, novembro de 2007. Disponível em: <http: //www.cileite.com.br/panorama/conjuntura13.html $>$. Acesso em: 17/3/2009.

KRUG, E. E. B. Sistema de produção de leite: identificação de benchmarking. Porto Alegre: Palloti, 2001. 245p. devem ser de boa qualidade, o que demonstra a necessidade de se introduzir conceitos sobre correto manejo sanitário dos animais e higiene durante a ordenha, em escolas rurais, considerando que os alunos serão futuros produtores.

Assim, para que haja melhoria na qualidade e produtividade do leite e o aumento do seu consumo e de seus derivados, foi pleiteada e garantida a prorrogação do projeto extensionista por mais vinte e um meses, visto a complexidade do caso e importância de obtenção de resultados positivos para o desenvolvimento agrário, econômico e social de toda a região.

LERNER, B. R.; LEI, D.L.M.; CHAVES, S. P.; FREIRE, R.D. O cálcio consumido por adolescentes de escolas públicas de Osasco, São Paulo. Revista de Nutrição, Campinas v.13, n. 1, jan./abr. 2000. Disponível em: < http: //www.scielo.br>. Acesso em: 27/3/2009.

OLIVEIRA, A. J. Leite: características, composição química, propriedades, obtenção higiênica, conservação e tratamento. Tecnologia dos produtos agropecuários. São Paulo: Nobel, 1997. p. 191-203.

TRONCO, V. M. Manual para inspeção da qualidade do leite. Santa Maria: UFSM, 2000. 115 p.

ZOCCAL, R. Estatísticas de Consumo. Agência de Informação Embrapa: agronegócio do Leite. Disponível em: <http://www.agencia.cnptia.embrapa.br/_Agencia8/ AG01/arvore/AG01_168_21720039245.html $>$. Acesso em: 25/3/2009.

Texto recebido em 30 de março de 2009.

Texto aprovado em 17 de abril de 2009. 